\title{
Aspiration of foreign bodies that allow air passage through
}

\author{
Demet Can $^{1}$, Ozge Yilmaz ${ }^{2 *}$, Suna Asilsoy ${ }^{1}$, Saniye Gulle ${ }^{1}$, Hasan Yuksel ${ }^{2}$ \\ ${ }^{1}$ Department of Pediatric Allergy, Behcet Uz Children's Hospital, Izmir, Turkey; \\ ${ }^{2}$ Department of Pediatric Allergy and Pulmonology, Medical Faculty, Celal Bayar University, Manisa, Turkey. \\ Email: *oyilmaz 76@,hotmail.com
}

Received 6 September 2011; revised 17 October 2011; accepted 3 November 2011.

\begin{abstract}
Foreign body aspiration is commonly encountered in children. In cases that foreign body does not disturb respiratory physiology, clinical and radiological diagnosis may be delayed leading to severe complications. Four cases with aspiration of a foreign body not obstructing ventilation and without typical clinical and radiological findings are discussed.
\end{abstract}

Keywords: Foreign Body; Aspiration; Children; Ventilation; Air Passage

\section{INTRODUCTION}

Foreign body aspiration is commonly encountered in children particularly less than three years age. Fourty eight patients were diagnosed with foreign body in the Emergency department of our hospital in 2005 and foreign body was removed in 44 . Among $50 \%$ these cases, foreign body was located in left main bronchus. History was suggestive in $67 \%$ of the children while radiological findings supported the diagnosis in $83 \%[1,2]$.

Although history and radiological findings leads to early diagnosis in many cases, delay in diagnosis leading to severe complications is not rare either. Delay is especially prominent when the foreign body does not cause airway obstruction therefore not disturb respiratory physiology. Four cases with aspiration of a foreign body that allow passage of air and therefore not obstructing ventilation and not causing significant clinical and radiological findings are discussed below.

\section{CASE REPORT}

\subsection{Case 1}

Twelve year old boy followed up for allergic asthma for three years had presented with cough and dyspnea to the Emergency department a month ago. He had been playing with a pen cap before the symptoms started and told this to the Emergency Department physician. Physical findings during that visit included bilateral ronchi, pro- longed expirium and tachpnea. His findings disappeared in short time after initiation of bronchodilator treatment. Both the chest X-ray and lung CT did not suggest foreign body aspiration. Upon fast resolution of clinical findings and absence of radiological findings, diagnosis of foreign body aspiration was ruled out and he was discharged with the diagnosis of an acute asthma exacerbation. However, the patient reported coughing up the pen cap three weeks after discharge. The foreign body that was coughed up had a hole inside that allowed air passage.

\subsection{Case 2}

Thirteen year old boy was hospitalized with symptoms of fever, productive cough and dyspnea. Past history revealed hospitalization due to pneumonia and pleuritis one year ago. Respiratory sounds were absent on the left side and decreased on the right side of the chest. Bronchial (tubular) breath sounds and fine rales were heard. White blood cell count (WBC) was increased to $21,900 / \mathrm{mm}^{3}$ and erythrocyte sedimentation rate was 70 $\mathrm{mm} /$ hour. CRP was 15.5 . Plain chest radiography suggested bronchiectasis and lung CT demonstrated left sided bronchiectasis (Figure 1). Immunoglobulin levels, tuberculin skin test and alphal anti-trypsin levels were normal. Sputum and blood cultures failed to reveal an infectious etiology. A piece of a whistle was seen in the left main bronchus during bronchoscopy and removed. The parents then remembered that he had had sudden cough and cyanosis when trying to blow out the plastic part inside the whistle. They had presented to a health care center where plain chest radiography was found to be normal. The foreign body that was removed allowed air passage at the midline.

\subsection{Case 3}

Two year old girl was hospitalized three times before she was sent for rigid bronchoscopy to another hospital. First hospitalization was at the age of 13 months with the diagnosis of acute bronchiolitis when she first had fever 


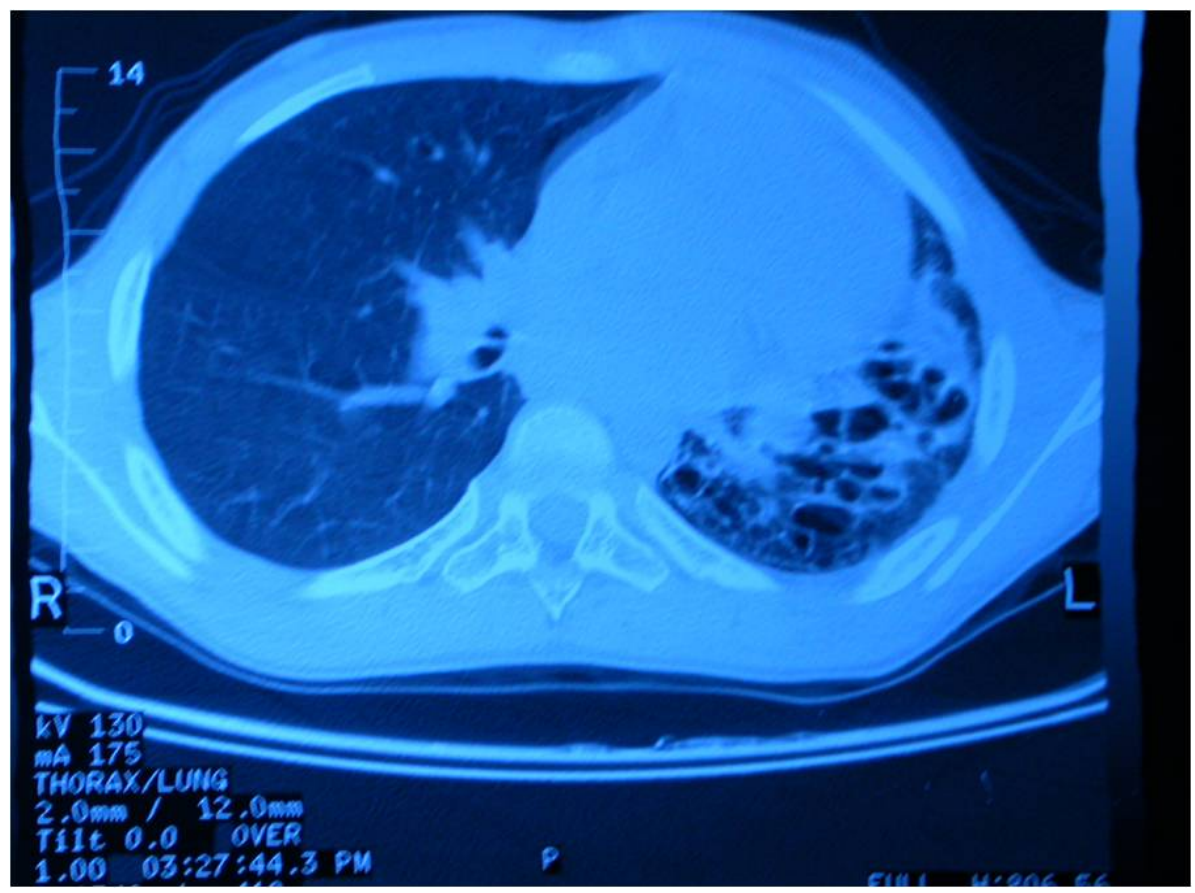

Figure 1. Lung CT of case 2 demonstrating left sided bronchiectasis.

and dyspnea. Past history revealed hospitalization in another hospital for a week at the age of 36 days with the diagnosis of bronchopneumonia. Body weight was 10.7 $\mathrm{kg}(50 \%-75 \%$, height was $74 \mathrm{~cm}(50 \%-75 \%)$. She had tachycardia (162/minute), tachypnea (48/minute). Subcostal, intercostal retractions and prolonged expiration time were detected. Bilateral hyperaeration was noticed in the plain radiograph (Figure 2). Nebulized salbutamol was administered and she was discharged on fifth day of hospitalization upon resolving of the clinical findings. She was hospitalized seven months after this because she had had four wheezing episodes after the first hospitali-

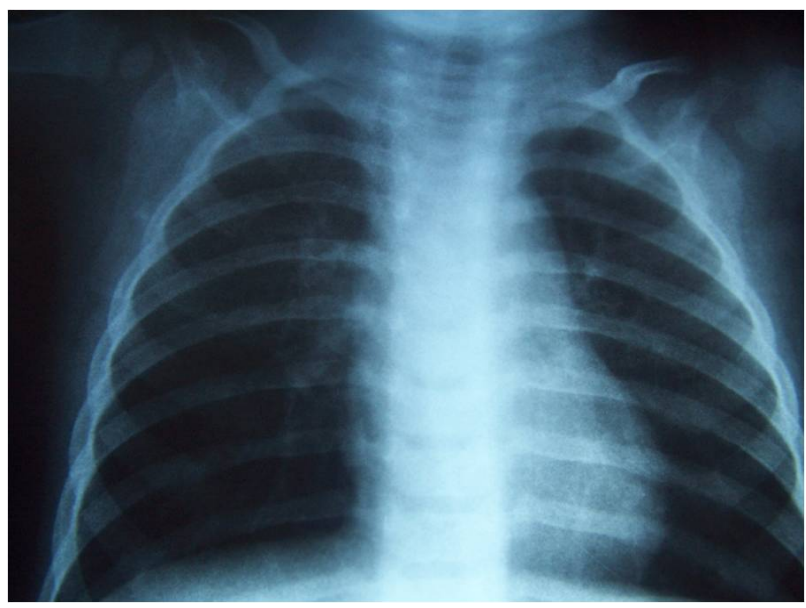

Figure 2. Bilateral hyperaeration in the plain chest radiograph of case 3 . zation. Lung auscultation revealed rhonchi. Serum levels of immunoglobulins, sweat chloride test and gastroesophageal reflux scintigraphy was normal. Plain chest radiography was normal but thorax $\mathrm{CT}$ was taken due to the persistence of auscultation findings on right side of chest. Focal area of atelectasis was detected on right lower lobe. Radiological findings were not found consistent with foreign body aspiration by the Radiologists. She was discharged and was started on inhaled steroids. Two months later, she was hospitalized again upon persistence of auscultation findings on the right side of chest. AngioCT revealed focal areas of narrowing in right middle and lower lobes that were interpreted in favor of bronchomalacia. Therefore the patient was sent to another center for rigid bronchoscopy during which a foreign body (plastic flower) was removed from right lung.

\subsection{Case 4}

Fourteen year old boy presented with cough. He had been hospitalized for treatment of lower respiratory tract infection many times for the last 6 months. Past medical and family histories were non-remarkable. Physical examination revealed bilateral fine rales and rhonchi that were more pronounced in the right lower lung fields. Complete blood count and serum biochemistry were normal. Immunoglobulin A, G and $\mathrm{M}$ values were in the normal ranges. Plain chest radiography demonstrated infiltration in the right middle lobe. Lung CT revealed right middle and lower lobe infiltration and mediastinal 
shift. Bronchial wall irregularity was seen in the segmental bronchus of the right upper lobe anterior segment and main bronchus of the right middle lobe. Flexible fiberoptic bronchoscopy was performed for diagnostic purposes and foreign body lodged right below enterance of right middle lobe was suspected. Pediatric surgery was called in during the same session for rigid bronchoscopy since the patient had received general anesthesia. They could not reach the foreign body in that session and interpreted the image as granulation tissue. However, upon questioning of the family again for foreign body aspiration, the child remembered swallowing a plastic pencil tip seven years ago. They had presented to a physician but aspiration was not thought at that time because plain radiography was normal. Upon this information, rigid bronchoscopy was performed once again and the foreign body was removed (Figure 3).

\section{DISCUSSION}

Foreign body aspiration that is most commonly encountered in pre-school children, is a life threatening emergency, however, it may go unrecognized for prolonged periods of time due to vague clinical and radiological findings in some cases $[3,4]$. Considering that most commonly aspirated foreign bodies in children include organic material including peanuts not causing complete obstruction, careful evaluation is especially important for prompt diagnosis [3].

Aspiration of a foreign body may result in immediate choking when lodged in larynx or may lead to complete obstruction of air entry into a lung segment [5,6]. Retained foreign body in the airway leads to local mechanical effects, chemical reactions and inflammation. An animal study has demonstrated that initial reaction to the presence of foreign body in the airway is polymerphonuclear leukocyte infiltration and edema which is followed by mononuclear leukocyte and macrophage infiltration. These findings have been interpreted as initiation of acute inflammation as early as three days after aspiration and progression to chronic inflammation as

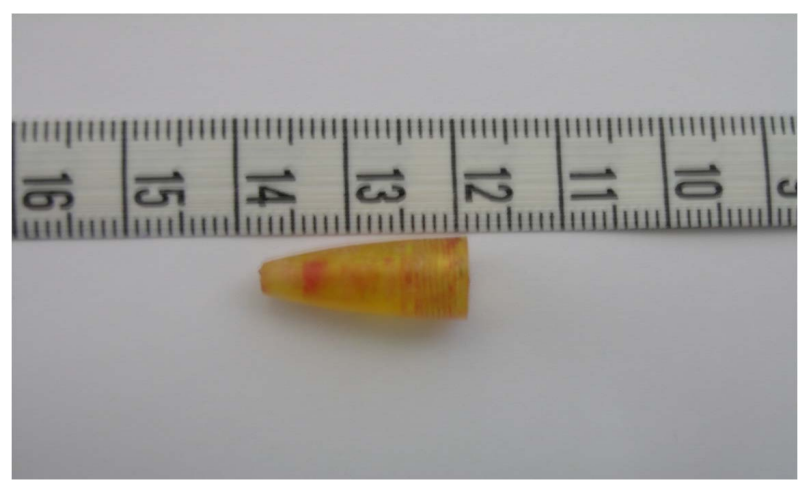

Figure 3. Foreign body aspirated by case 4 . early as ten days. Moreover, bronchiectatic changes were observed when a month has passed after aspiration [7].

Presenting symptoms of foreign body aspiration may vary from vague to specific and include cough, wheeze, dyspnea and fever. Physical examination may reveal focal wheezing or decreased air entry but the findings may also reveal generalized wheezing or it may be completely normal [8]. Similarly plain radiographs of chest may reveal unilateral hyperinflation, atelectasis, consolidation or mediastinal shift if there is complete obstruction of airflow by the foreign body or they may be normal especially if there is no obstruction to airflow [5, 8]. Therefore, it is impossible to exclude diagnosis of foreign body aspiration with a normal radiograph [9]. In the presented cases, two of them had findings suggestive of bronchiectasis in plain radiographs which was confirmed by computerized tomography (CT). Two patients had normal plain radiography findings. One of the latter two had persistent findings on physical examination and therefore underwent CT that demonstrated focal atelectasis.

Diagnosis of foreign body aspiration is usually suggested with clinical history and radiological findings [3]. Foreign body is encountered only in $5 \%$ of cases that undergo flexible bronchoscopy without a prior suspicion of aspiration [3,5]. Considering that early removal of aspirated foreign bodies is necessary to avoid the pathological progress from inflammation that initiates at third day to development of bronchiectasis after 30 days, high suspicion even in cases with vague clinical or radiological findings is required $[7,8]$. Four cases presented in this report highlight the importance of aspiration of foreign bodies that do not cause complete obstruction since these cases may easily be overlooked and bronchoscopy may be delayed. In three of these cases clinical findings were not suggestive of foreign body aspiration due to the nature of the foreign body that allowed air passage. One patient was lucky to cough up the aspirated material but the other three presented with bronchiectasis, pneumonia or bronchoconstriction findings [8]. Although the clinical findings may be milder without complete airway obstruction, induction of inflammation is still expected to progress thus making early and prompt diagnosis essential for prevention of complications. Therefore, high clinical suspicion and use of flexible bronchoscopy as the initial technique of evaluation in patients with suspected foreign body aspiration is prompted. Moreover, Flexible bronchoscopy provides detailed information about the nature and localization of the foreign body as well as the characteristics of airway mucosa [3].

Detection of a foreign body aspirated into the airway should be followed by removal as soon as possible to prevent the inflammatory reaction and development of 
granulation tissue [4]. Although, flexible bronchoscopy is very often used for evaluation of airway in suspected cases of foreign body aspiration, rigid bronchoscopy remains the method of choice for removal due to the wide working channel. There are a limited number of reports of foreign body removal by flexible bronchoscopy [3]. With the exception of the case who has coughed up the foreign body, in all three cases the foreign body was removed with rigid bronchoscopy.

In conclusion, diagnosis of foreign body aspiration was delayed in all the cases presented in this report due to the non-obstructing nature of the foreign body. Air passage through the foreign body delayed appearance of radiological clues. These cases were reported to emphasize the importance of early consideration of bronchoscopy in cases with persistent findings even in the absence of supporting clinical and radiological clues of foreign body aspiration.

\section{REFERENCES}

[1] Cotton, R.T. and Rutter, M.J. (2006) Foreign body aspiration. In: Chernick, V., Boat, T.F., Wilmott, R.W., et al., Eds., Kendig's Disorders of Respiratory Tract in Children, 5th Edition, Saunders, Philadelphia, 610-615.

[2] Ağın, H., Büber, B., Apa, H., Asilsoy, S., Gulez, N. and Atlıhan, F. (2007) Çocuklarda yabancı cisim aspirasyonu. Ege Pediatri Bülteni, 14, 101-106.

[3] Ramírez-Figueroa, J.L., Gochicoa-Rangel, L.G., RamírezSan Juan, D.H. and Vargas, M.H. (2005) Foreign body removal by flexible fiberoptic bronchoscopy in infants and children. Pediatric Pulmonology, 40, 392-397. doi:10.1002/ppul.20242

[4] Ibrahim Sersar, S., Hamza, U.A., AbdelHameed, W.A. AbulMaaty, R.A., Gowaeli, N.N., Moussa, S.A., et al. (2005) Inhaled foreign bodies: Management according to early or late presentation. European Journal of CardioThoracic Surgery, 28, 369-374. doi:10.1016/j.ejcts.2005.05.013

[5] Karakoc, F., Cakir, E., Ersu, R., Uyan, Z.S., Colak, B., Karadag, B., et al. (2007) Late diagnosis of foreign body aspiration in children with chronic respiratory symptoms. International Journal of Pediatric Otorhinolaryngology, 71, 241-246. doi:10.1016/j.ijporl.2006.10.006

[6] Bloom, D.C., Christenson, T.E., Manning, S.C., Eksteen, E.C., Perkins, J.A., Inglis, A.F., et al. (2005) Plastic laryngeal foreign bodies in children: A diagnostic challenge. International Journal of Pediatric Otorhinolaryngology, 69, 657-662. doi:10.1016/j.ijporl.2004.12.006

[7] Yildizeli, B., Zonuzi, F., Yuksel, M., Kodalli, N., Cakalagaoglu, F. and Kullu, S. (2002) Effects of intrabronchial foreign body retention. Pediatric Pulmonology, 33, 362-367. doi:10.1002/ppul.10116

[8] Daines, C.L., Wood, R.E. and Boesch, R.P. (2002) Foreign body aspiration: An important etiology of respiratory symptoms in children. Journal of Allergy and Clinical Immunology, 121, 1297-1298. doi:10.1016/j.jaci.2008.03.014

[9] Pinzoni, F., Boniotti, C., Molinaro, S.M., Baraldi, A. and Berlucchi, M. (2007) Inhaled foreign bodies in pediatric patients: Review of personal experience. International Journal of Pediatric Otorhinolaryngology, 71, 1897 1903. doi:10.1016/j.ijporl.2007.09.002 